\title{
Single pass, THz spectral range free-electron laser driven by a photocathode hybrid rf linear accelerator
}

\author{
Yu. Lurie, ${ }^{*}$ A. Friedman, ${ }^{\dagger}$ and Y. Pinhasi ${ }^{\ddagger}$ \\ Ariel University, Ariel \\ (Received 14 January 2015; published 6 July 2015)
}

\begin{abstract}
A single pass, THz spectral range free-electron laser (FEL) driven by a photocathode hybrid rf-LINAC is considered, taking the Israeli THz FEL project developed in Ariel University as an example. Two possible configurations of such FEL are discussed: an enhanced coherent spontaneous emission FEL, and a prebunched FEL utilizing periodically modulated short electron beam pulses. A general study of the FEL configurations is carried out in the framework of a space-frequency approach, realized in WB3D numerical code. The configurations are studied and compared based on preliminary parameters of a drive hybrid rf-LINAC gun under development in University of California, Los Angeles.
\end{abstract}

DOI: 10.1103/PhysRevSTAB.18.070701

PACS numbers: 41.60.Cr, 52.59.Rz

\section{INTRODUCTION}

Tera-Hertz electromagnetic radiation is currently of interest for many applications such as basic science, medical and biological applications, spectrometry, remote detection, and more. Modern $\mathrm{THz}$ free-electron lasers (FELs) often can fit a "table-top" size. They are able to provide intense coherent radiation over a wide range of $\mathrm{THz}$ frequencies. In the present work, we consider a single pass FEL (no resonant cavity is present) for production of $\mathrm{THz}$ radiation. The FEL is driven by short electron pulses, produced by a photo-injector rf-LINAC gun. Note that a hybrid rf-LINAC structure including both standing and traveling waves sections enables production of short (picosecond or sub-picosecond scale long) electron beam pulses with a controlled energy chirping. The Israeli rf-LINAC THz FEL project under development in Ariel university is considered here as an example of such radiation source [1]. The basic operational parameters of the FEL are given in Table I. Two possible configurations of the FEL are considered and compared in this work: an enhanced coherent spontaneous emission (ECSE) FEL discussed in [2], and a prebunched FEL utilizing periodically modulated short electron beam pulses.

ECSE FEL [2] (called in the publication as an "enhanced super-radiance" FEL) utilizes a constructive energy-phase correlation [3], which results in an enhancement of undulator coherent spontaneous emission (CSE) of short (a radiation wavelength long or shorter) electron pulses. Energy chirping

\footnotetext{
*ylurie@ariel.ac.il

aharonfr@ariel.ac.il

yosip@ariel.ac.il
}

Published by the American Physical Society under the terms of the Creative Commons Attribution 3.0 License. Further distribution of this work must maintain attribution to the author(s) and the published article's title, journal citation, and DOI. is a well-known technique used in accelerator physics for density compression of electron pulses. In an ECSE FEL, it is suggested to cause a longitudinal density compression of short electron beam pulses just inside the FEL interaction region, i.e., inside the undulator or wiggler. Note that a proper energy chirping enables one to improve the emitting electrons phase-matching as well. It was shown that both effects (density compression and a better phase-matching) can provide a strong enhancement of CSE [2].

Electron beam prebunching or modulation is a technique well known and used in radiation sources. A THz frequency periodic modulation can be introduced in relativistic short electron beam pulses by means of a proper temporal shaping of photocathode laser pulse using a short pulsed frequency chirped laser [4]. The technique was demonstrated to enable production of modulated beam pulses of a desired modulation frequency. It even provided a controlled number of modulation oscillations. Following [4], application of this modulation technique to THz FEL is considered here.

In order to simulate and compare between these FEL configurations, the 3D space-frequency approach [5] is

TABLE I. Operational parameters for the THz FEL.

\begin{tabular}{ll}
\hline \hline Accelerator & \\
Type: & Hybrid photo-injector rf-LINAC \\
Electron beam energy: & $E_{k}=5.5 \mathrm{MeV}$ \\
Pulse duration: & $T_{b} \gtrsim 0.1 \mathrm{ps}$ \\
Beam radius: & $R_{b} \approx 1 \mathrm{~mm}$ \\
Bunch charge: & $Q_{b}=30-500 \mathrm{pC}$ (short single pulse) \\
& $Q_{b} \lesssim 2 \mathrm{nC}$ (long pulse) \\
Wiggler & $B_{w}=2 \mathrm{kG}$ \\
Magnetic induction: & $\lambda_{w}=25 \mathrm{~mm}$ \\
Period: & \\
Waveguide & \\
Rectangular waveguide: & $15 \times 10 \mathrm{~mm}^{2}$ \\
Radiation & \\
Synchronism frequency: & $f_{s} \approx 2.96 \mathrm{THz}\left(\lambda_{s} \approx 100 \mu \mathrm{m}\right)$ \\
\hline \hline
\end{tabular}


applied. The method is based on an expansion of the total electromagnetic field in terms of transverse eigenmodes of the medium (free-space or waveguide) in which the field is excited and propagates. The interaction between the electromagnetic field and the gain medium is fully described by a set of coupled equations, expressing the evolution of mode amplitudes along the interaction region. The model was realized in a numerical code WB3D and has been successfully applied to the analysis of various effects in FEL devices [6-12].

\section{THE APPROACH}

The model is based on the space-frequency $3 \mathrm{D}$ approach described in more detail in [5]. A short explanation of the method is given here for convenience.

\section{A. Electromagnetic field}

Electromagnetic field emitted by a wiggling electron pulse in an uniform waveguide is considered in the positive-defined frequency domain in terms of a summation over the transverse eigenmodes of the cold waveguide in which the radiation is excited and propagates:

$$
\begin{aligned}
& \mathbf{E}(\mathbf{r}, t)=\sum_{q} \Re\left[\int_{0}^{\infty} \tilde{C}_{q}(z, f) \tilde{\mathcal{E}}_{q}\left(\mathbf{r}_{\perp}\right) e^{-j\left(k_{z q} z-2 \pi f t\right)} \mathrm{d} f\right] \\
& \mathbf{H}(\mathbf{r}, t)=\sum_{q} \Re\left[\int_{0}^{\infty} \tilde{C}_{q}(z, f) \tilde{\mathcal{H}}_{q}\left(\mathbf{r}_{\perp}\right) e^{-j\left(k_{z q} z-2 \pi f t\right)} \mathrm{d} f\right]
\end{aligned}
$$

where $\tilde{\mathcal{E}}_{q}\left(\mathbf{r}_{\perp}\right)$ and $\tilde{\mathcal{H}}_{q}\left(\mathbf{r}_{\perp}\right)$ are the transverse electric and magnetic profiles of a waveguide eigenmode number $q, k_{z q}$ is the longitudinal wave number of the mode, $\mathbf{r}_{\perp} \equiv\{x, y\}$ is the transverse position, and $z$ is the longitudinal axis of the beam propagation. The mode amplitude $\tilde{C}_{q}(z, f)$ can be found by solving the excitation equation:

$$
\begin{aligned}
\frac{d}{d z} C_{ \pm q}(z, f)= & \mp \frac{1}{2 \mathcal{N}_{q}(f)} e^{ \pm j k_{z q} z} \\
& \times \iint\left[\tilde{\mathbf{J}}_{\perp}(\mathbf{r}, f)+\hat{\mathbf{z}} \tilde{J}_{z}(\mathbf{r}, f)\right] \cdot \tilde{\mathcal{E}}_{ \pm q}{ }^{*}\left(\mathbf{r}_{\perp}\right) \mathrm{d} \mathbf{r}_{\perp}
\end{aligned}
$$

where

$$
\mathcal{N}_{q}(f)=\iint\left[\tilde{\mathcal{E}}_{q \perp} \times \tilde{\mathcal{H}}_{q \perp}^{*}\right] \cdot \hat{\mathbf{z}} \mathrm{d} \mathbf{r}_{\perp}
$$

is the power normalization of mode $q$, and $\tilde{\mathbf{J}}(\mathbf{r}, f)$ is the frequency-domain current density of a drive electron beam.

The total energy flux of the emitted electromagnetic radiation is

$$
W_{\mathrm{EM}}(z)=\int_{0}^{\infty} \frac{\mathrm{d} W(z)}{\mathrm{d} f} \mathrm{~d} f
$$

where

$$
\begin{aligned}
\frac{\mathrm{d} W(z)}{\mathrm{d} f} & =\frac{1}{2} \Re\left\{\iint\left[\tilde{\mathbf{E}}(\mathbf{r}, f) \times \tilde{\mathbf{H}}^{*}(\mathbf{r}, f)\right] \cdot \hat{\mathbf{z}} \mathrm{d} x \mathrm{~d} y\right\} \\
& =\frac{1}{2} \sum_{q}\left|C_{q}(z, f)\right|^{2} \Re\left\{\mathcal{N}_{q}(f)\right\}
\end{aligned}
$$

defines the radiation energy flux spectral density. The radiation power is given by $P_{\mathrm{EM}}(z)=W_{\mathrm{EM}}(z) / \tau_{\mathrm{sp}}(z)$, where $\tau_{\mathrm{sp}}(z)=z\left|1 / v_{\mathrm{gr}}-1 / v_{z}\right|$ is the slippage time of the radiation $\left(v_{z}\right.$ is the average beam propagation velocity, and $v_{\mathrm{gr}}$ is the group velocity of the emitted wave packet). Note that backward going waves are out of synchronism with drive electron beam in the FEL configurations considered, and are therefore neglected. In our case, a simple initial condition $C_{q}(z=0, f)=0$ is applied for a numerical solution of the excitation equations (2).

\section{B. Electron beam dynamics}

Current density of the drive electron beam is presented as a summation over a number of charged, point particles:

$$
\begin{aligned}
\mathbf{J}(\mathbf{r}, t) & =-\sum_{i} q_{i} \mathbf{v}_{i} \delta\left(\mathbf{r}_{\perp}-\mathbf{r}_{\perp_{i}}\right) \delta\left[z-z_{i}(t)\right] \\
& =-\sum_{i} q_{i}\left(\frac{\mathbf{v}_{i}}{v_{z_{i}}}\right) \delta\left(\mathbf{r}_{\perp}-\mathbf{r}_{\perp_{i}}\right) \delta\left[t-t_{i}(z)\right]
\end{aligned}
$$

where $q_{i}$ is the charge of the $i$ th particle in the simulation and $t_{i}(z)$ is the time it takes for the particle to arrive at a position $z$, which is a function of the time $t_{0 i}$ when a particle entered at $z=0$, and of the instantaneous longitudinal velocity $v_{z i}(z)$ :

$$
t_{i}(z)=t_{0_{i}}+\int_{0}^{z} \frac{1}{v_{z_{i}}\left(z^{\prime}\right)} \mathrm{d} z^{\prime} .
$$

After substitution of Fourier transform of the current density (6) in Eq. (2), the last can be rewritten in the form:

$$
\begin{aligned}
\frac{d}{d z} C_{ \pm q}(z, f)= & \pm \frac{1}{\mathcal{N}_{q}(f)} e^{ \pm j k_{z q} z} \\
& \times \sum_{i} q_{i} e^{-j 2 \pi f t_{i}(z)} \frac{\mathbf{v}_{i} \cdot \tilde{\mathcal{E}}_{ \pm q}^{*}\left(\mathbf{r}_{\perp_{i}}\right)}{v_{z_{i}}}
\end{aligned}
$$

The velocity of each particle, in the presence of an electric $\mathbf{E}(\mathbf{r}, t)$ and a magnetic $\mathbf{B}(\mathbf{r}, t)=\mu \mathbf{H}(\mathbf{r}, t)$ fields, is found from the Lorentz force equation: 


$$
\frac{\mathrm{d}}{\mathrm{d} t}\left(\gamma_{i} \mathbf{v}_{i}\right)=-\frac{e}{m}\left[\mathbf{E}\left(\mathbf{r}_{i}, t\right)+\mathbf{v}_{i} \times \mathbf{B}\left(\mathbf{r}_{i}, t\right)\right]
$$

where $e$ and $m$ are the electron charge and mass, respectively. The fields represent the total (DC and AC) forces operating on the particle, and include the self-field due to space-charge. The Lorentz relativistic factor $\gamma_{i}$ of each particle is found from the equation for kinetic energy:

$$
\frac{\mathrm{d} \gamma_{i}}{\mathrm{~d} t}=-\frac{e}{m c^{2}} \mathbf{v}_{i} \cdot \mathbf{E}\left(\mathbf{r}_{i}, t\right)
$$

where $c$ is the speed of light. Equations (9) and (10) can be rearranged by replacing the time derivative $\frac{\mathrm{d}}{\mathrm{d} t}=v_{z_{i}} \frac{\mathrm{d}}{\mathrm{d} z}$ such that the coordinate of the propagation axis $z$ becomes the independent variable.

Equations (1), (3) and (7)-(10) form a closed set of nonlinear equations, which enable a self-consistent solution for both radiation field and beam trajectory. Correctness of the solution can be controlled by checking the energy conservation along the beam propagation:

$$
W_{b}(z=0) \approx W_{\mathrm{EM}}(z)+W_{b}(z)
$$

where the potential energy of the space-charge forces is neglected, and the beam pulse kinetic energy is given by

$$
W_{b}(z)=\frac{m_{0} c^{2}}{e} \sum_{i}\left|q_{i}\right|\left[\gamma_{i}(z)-1\right] \approx \frac{Q_{b}}{e} E_{b}
$$

( $Q_{b}=\sum_{i}\left|q_{i}\right|$ and $E_{b}$ are the beam pulse charge and the energy, relatively). Mention that the relation $W_{\mathrm{EM}}\left(L_{w}\right) / W_{b}(z=0)$ between the output radiation energy flux to the initial beam energy gives an evaluation of the FEL radiation extraction efficiency.

\section{Initial charge distribution}

The drive electron beam is introduced in the simulations by an initial distribution of the particles at the point $z=0$. In ECSE FELs, a coherent emission of a short (in terms of the radiation wavelength) electron bunch takes place. An initial random Gauss distribution of equally charged particles is used for simulations of this effect. Such model enables an accurate simulation of the emission process by means of a relatively small number of the particles in a simulation.

In current density given by (6), each "macro"-particle actually represents $Q_{b} /\left(e N_{q}\right)$ electrons of the beam, where $Q_{b}$ is the beam charge and $N_{q}$ is the number of particles in a simulation. In contrast with shot noise of randomlydistributed electrons, all the electrons a particle "consists of" emit coherently, producing a $Q_{b} /\left(e N_{q}\right)$ times stronger radiation than a spontaneous emission of the real beam. To overcome this limitation, the numerical algorithm of Ref. [13] was also realized for the particle loading. In the algorithm, an initial time and a charge of each particle are randomly distributed according the beam current density profile, simulating the initial shot noise of a real electron beam in a wide range of frequencies (unified model of electron beam shot noise). The options of a Gaussian form beam current density profile (for simulations of a single pulse configuration), and of a beam pulse of a periodically modulated beam current density (for simulations of a prebunched beam configuration) were introduced in the algorithm.

\section{Space-charge fields}

The electromagnetic field of each charge in the beam influences the rest of the charges, disturbing their trajectories. These space-charge (SC) effects are important for the description of dynamics of a high charge density beams. This, for example, takes place in an ECSE FEL as a result of the bunch compression. Precise description of mutual electromagnetic interactions between relativistic charges arbitrary moving in a metal waveguide appears to be a complicated, hardly solvable problem. To simplify numerical evaluation of SC fields, the effect of the waveguide walls can be neglected and the "free-space" interaction can be used instead. To obtain an analytical expression for the field of a relativistic charge, one can consider the rest frame of the charge where the field is an electrostatic and is simply given in this approach by Coulomb law. This field can then be transformed back to the laboratory frame. The total space-charge field affecting a charge can be found by a superposition of the fields of each individual charge in the beam. This laboratory frame field is introduced in Eqs. (9), (10) in order to evaluate a distortion of the beam trajectory due to space-charge effects. The approach is similar to a relativistic "point-to-point" model of SC fields realized in commercial General Particle Tracer code [14].

\section{ECSE FEL}

Enhanced coherent spontaneous emission is obtained in FELs driven by short electron pulses (with length of about the emitted radiation wavelength, or shorter) which are subjected to a controlled energy chirp (energy modulation). Usually, spontaneous emission takes place if the time duration of the emitting electron bunch is about that of the radiation period. However energy chirp can give rise to a longitudinal density compression of electron pulses as they propagate along the wiggler. This longitudinal density compression, together with an improvement of the emitting electrons phase-matching (due to a proper energy chirping), enable a strong enhancement of CSE [2]. Short (a subpicosecond or a femtosecond scale long), high energychirping pulses of relativistic electron beam which are required in this scheme, can be produced by means of photocathode rf-LINAC by some phase desynchronization 


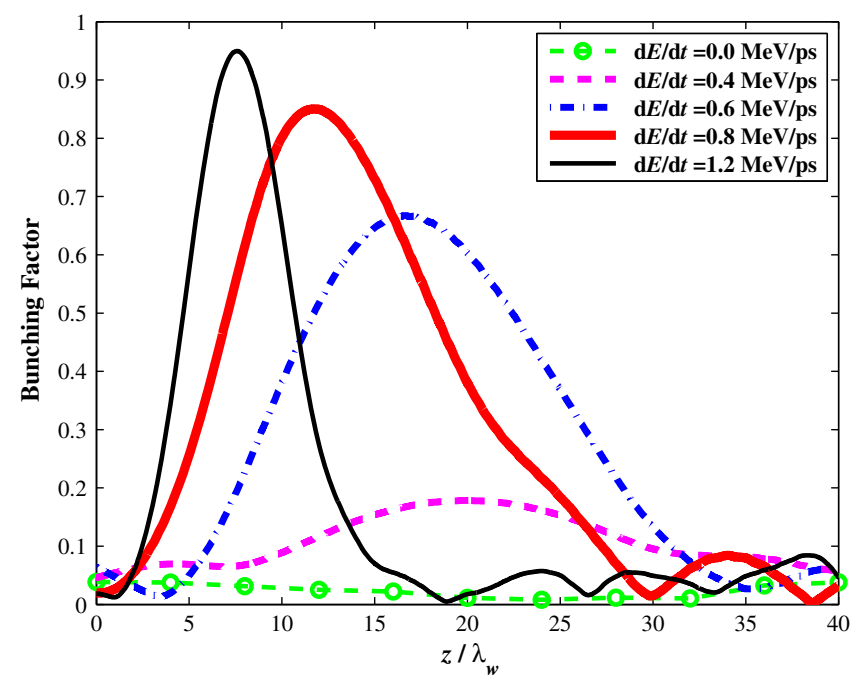

FIG. 1. Evolution of the bunching factor along the bunch trajectory in the ECSE FEL. The bunch charge $Q_{b}=30 \mathrm{pC}$, and the bunch duration $f_{s} T_{b} \approx 1$. Random Gauss initial distribution of $N_{q}=300$ charged particles.

of accelerated electron pulses relative to the accelerating electromagnetic wave in the rf-LINAC cavities. In a hybrid rf-LINAC structure [15] including both standing and traveling wave sections, desired phase desynchronization of electron pulses accelerated preliminary in the standing wave part can later be obtained in the following traveling wave section. Note that this is similar to the idea of the "phase-matching" section within a FEL beam line utilized in [3].

The basic operational parameters of THz ECSE FEL are given in Table I. In order to enable a flexible ECSE FEL

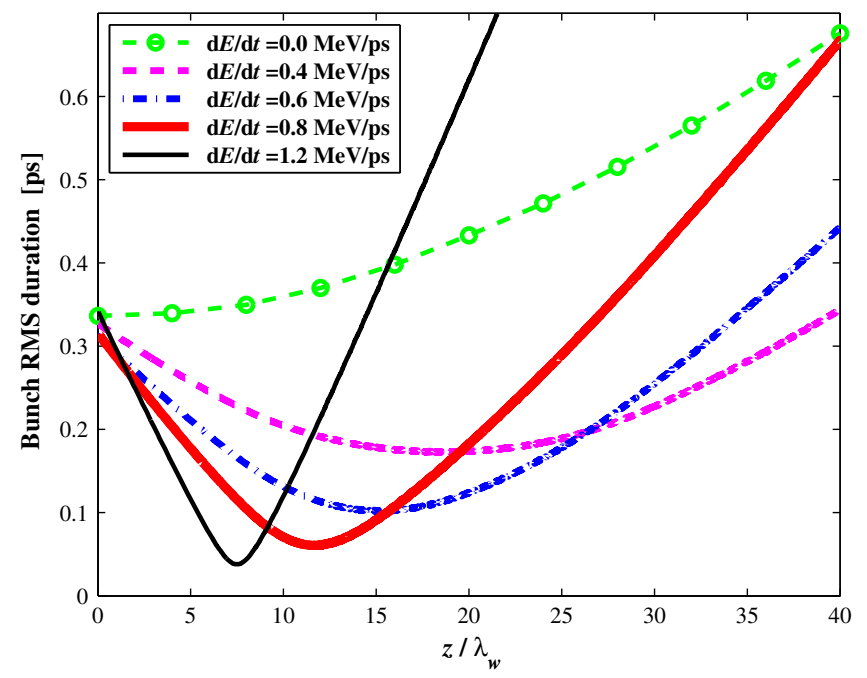

FIG. 2. Evolution of bunch RMS duration along the trajectory in the ECSE FEL. The bunch charge $Q_{b}=30 \mathrm{pC}$, and the bunch duration $f_{s} T_{b} \approx 1$. Random Gauss initial distribution of $N_{q}=$ 300 charged particles.
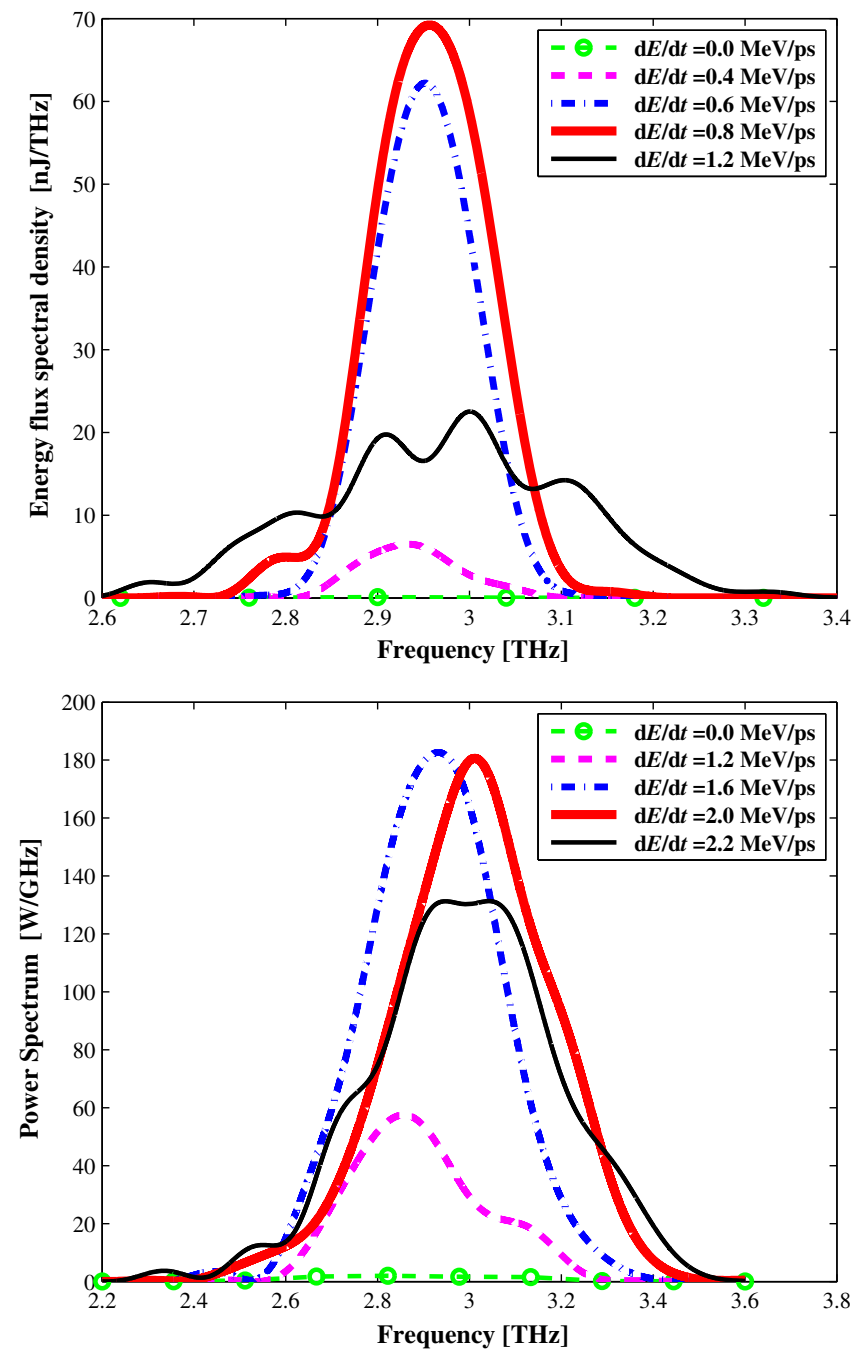

FIG. 3. Radiation spectrum emitted in the ECSE FEL. The bunch charge $Q_{b}=30 \mathrm{pC}$ (top) or $Q_{b}=300 \mathrm{pC}$ (bottom), and the bunch duration $f_{s} T_{b} \approx 1$. Random Gauss initial distribution of $N_{q}=300$ charged particles.

operation at wide range of energy chirping levels, a relatively long wiggler with $N_{w}=40$ periods is assumed. Figure 1 demonstrates evolution of the bunching factor obtained with $Q_{b}=30 \mathrm{pC}$ electron bunches propagating along the wiggler. The simulations were carried out with random Gauss initial distribution of $N_{q}=300$ charged particles. As seen from the figure, a proper energy chirp rate $\frac{d E}{d t} \gtrsim 0.6 \mathrm{MeV} / \mathrm{ps}$ can produce considerable beam bunching with a bunching factor of $70 \%$ or more. The effect is also illustrated by the evolution of the bunch RMS time duration shown in Fig. 2. Strong enhancement of coherent spontaneous emission arises mainly due to the kinematics of short, energy-chirped beam pulse and was discussed in more detail in [2]. Note that an energy chirp rate of $\frac{d E}{d t} \approx$ $0.6 \mathrm{MeV} / \mathrm{ps}$ with $T_{b} \approx 1 / f_{s} \sim 0.34$ ps pulses means a total relative energy spread of 

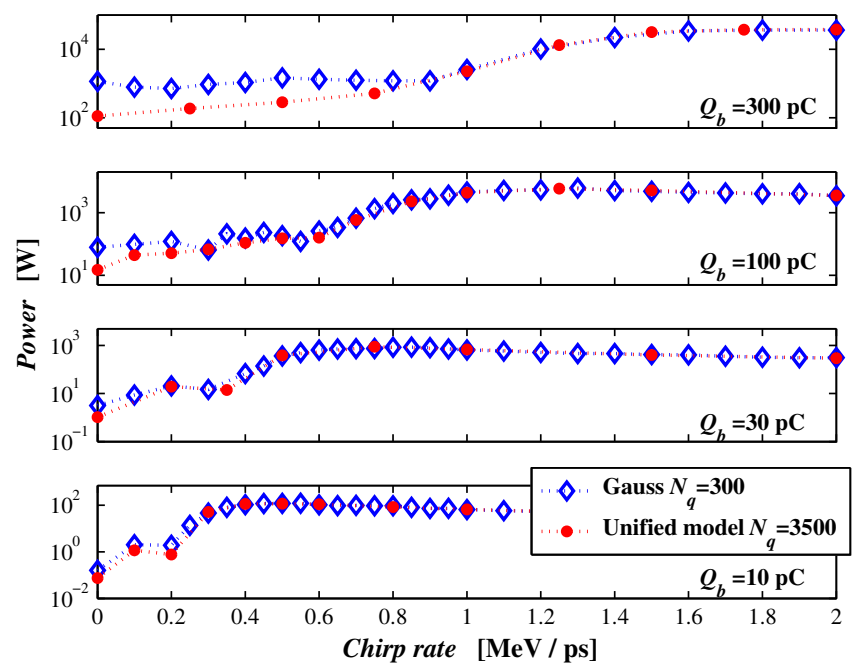

FIG. 4. ECSE FEL power dependence on the chirp rate (the bunch duration is $f_{s} T_{b} \approx 1$ ). Random Gauss initial distribution of $N_{q}=300$ charged particles, and unified model of electron beam shot noise $\left(N_{q}=3500\right)$ with a Gaussian form beam current density profile.

$$
\frac{\Delta E}{E_{b}}=\frac{1}{E_{b}} \frac{d E}{d t} T_{b} \approx 3.6 \%
$$

This high beam bunching results in a considerable growth of the radiation spectrum, as demonstrated in Fig. 3. A proper choice of the chirp rate can provide a remarkable increase in the total radiation power (about two orders of magnitude), which one can see in Fig. 4 where the total radiation power is given as a function of the chirp rate.

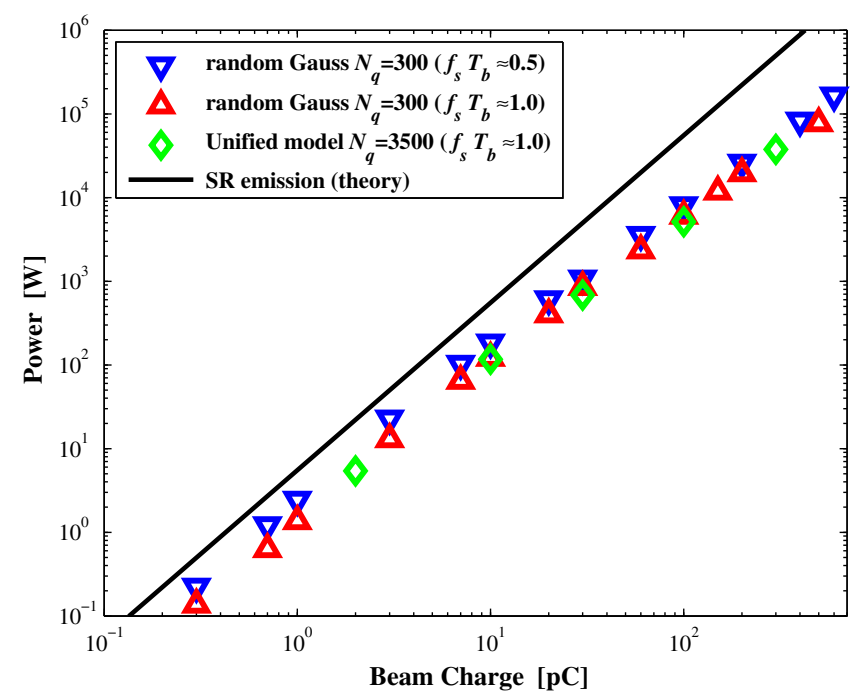

FIG. 5. ECSE FEL radiation power dependence on the beam charge. Random Gauss initial distribution of $N_{q}=300$ charged particles, and unified model of electron beam shot noise $\left(N_{q}=3500\right)$ with a Gaussian form beam current density profile.
The charge dependence of the total radiation power obtained at an "optimal" chirp rate (i.e., at the chirp rate resulting in the highest radiation power) is shown in Fig. 5. The total radiation power seems to behave like the square of the beam charge up to a charge of a few tens of picoCoulombs; at higher charges, the growing space-charge effects results in considerable reduction of the power. Note that proportionality of the radiation power with a charge squared is a well-known property of super-radiant emission [7]. Results of analytical expression for power of superradiant emission are also shown in the picture for comparison.

\section{PREBUNCHED BEAM THZ FEL}

Prebunched beam FEL utilizes an electron beam pulse, periodically modulated at a $\mathrm{THz}$ frequency equal to the frequency of the desired radiation. Such beam pulse can be obtained by a splitting of the photo-injector's optical laser beam. One partial beam is given a longer path, the beams are then unified on the photocathode's surface. Since the laser is frequency chirped, this results in beats of the intensity on the photocathode. The technique was demonstrated to enable production of modulated beam pulses of a desired modulation frequency, and even provides a control on a number of modulation oscillations [4]. Because of a long (multiple periods of the prebunching modulation) beam pulse, electron beams with a relatively low chirping or even monoenergetic electron beams should be used in this scheme. Due to the destructive effect of space-charge forces, a region of an effective beam-radiation interaction is limited and a wiggler of $N_{w}=20$ periods only was considered for this FEL configuration. An initial beam current was defined in the simulations by applying the

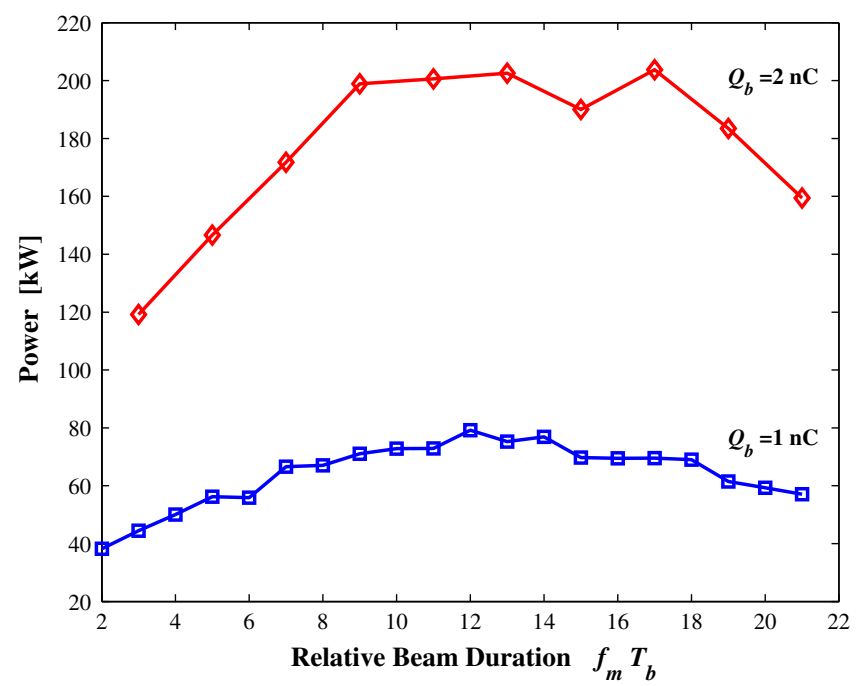

FIG. 6. Radiation power of a prebunched FEL as a function of the number of beam microbunches (relative beam duration $f_{m} T_{b}$ ). The modulation index and the modulation frequency are $m=50 \%$ and $f_{m}=3 \mathrm{THz}$, respectively. 

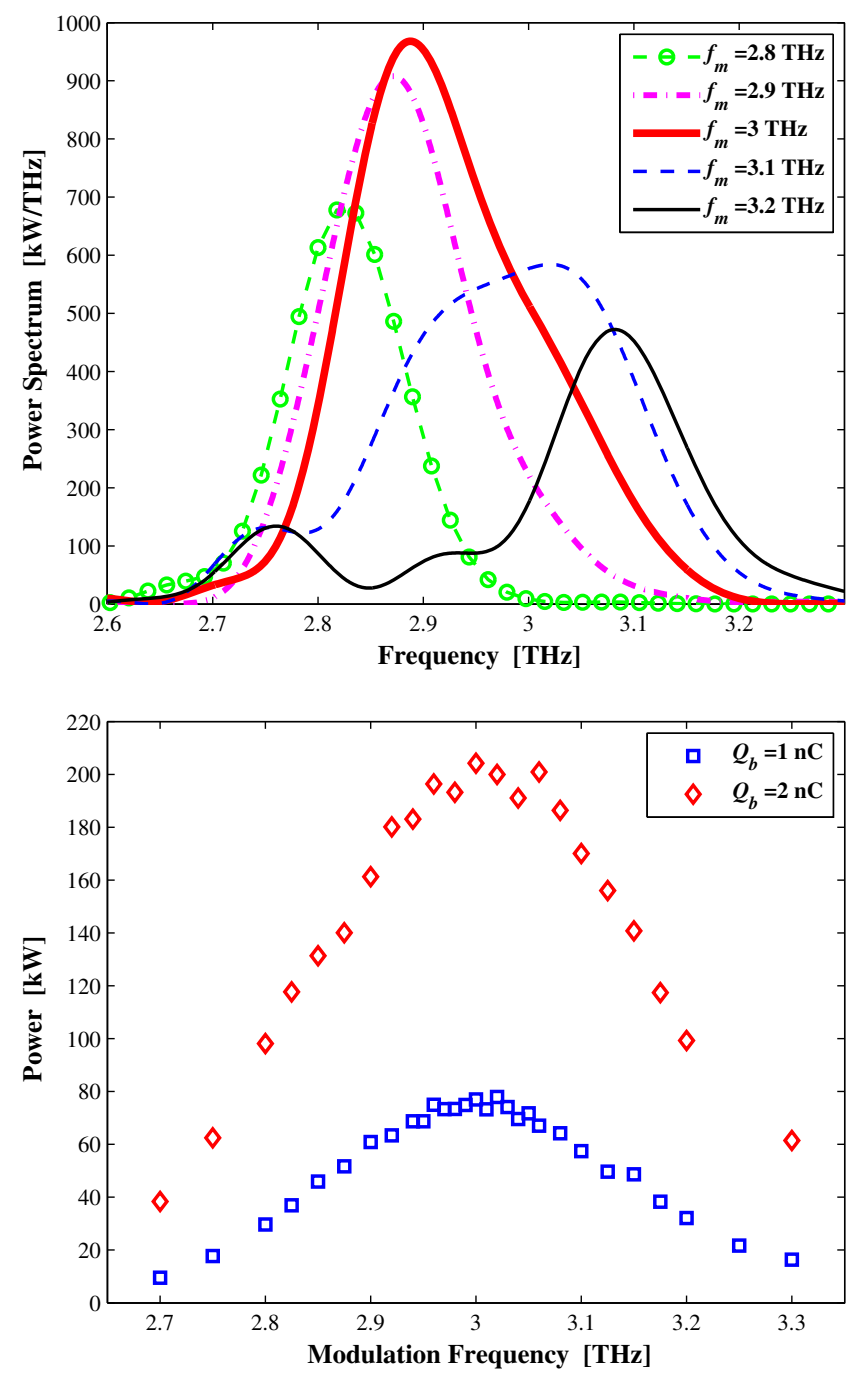

FIG. 7. Power spectrum of prebunched FEL ( $Q_{b}=2 \mathrm{nC}$, top), and the total power (bottom) in dependence on the modulation frequency $f_{m}$. The modulation index is $m=50 \%$.

unified model of electron beam shot noise, assuming a cosine-like beam current density. $N_{q}=3500$ particles were introduced in the simulations to present a modulated beam pulse.

At the first step, an FEL driven by a monoenergetic prebunched beam pulse was examined. Figure 6 presents a power of the radiation emitted by a prebunched FEL as a function of the number of beam microbunches, or a relative beam duration $N_{m} \equiv f_{m} T_{b}$. The modulation index is $m=50 \%$, and the modulation frequency is $f_{m}=3 \mathrm{THz}$. The power is shown for both $Q_{b}=1 \mathrm{nC}$ and $Q_{b}=2 \mathrm{nC}$ beam pulse charges. Both curves are similar; besides small variations, the power slowly grows with a relative beam duration up to a plateau at $f_{m} T_{b} \approx 10-17$, where it seems to be approximately constant. In the following simulations, beam pulses with the relative duration of $f_{m} T_{b}=11$ were considered.

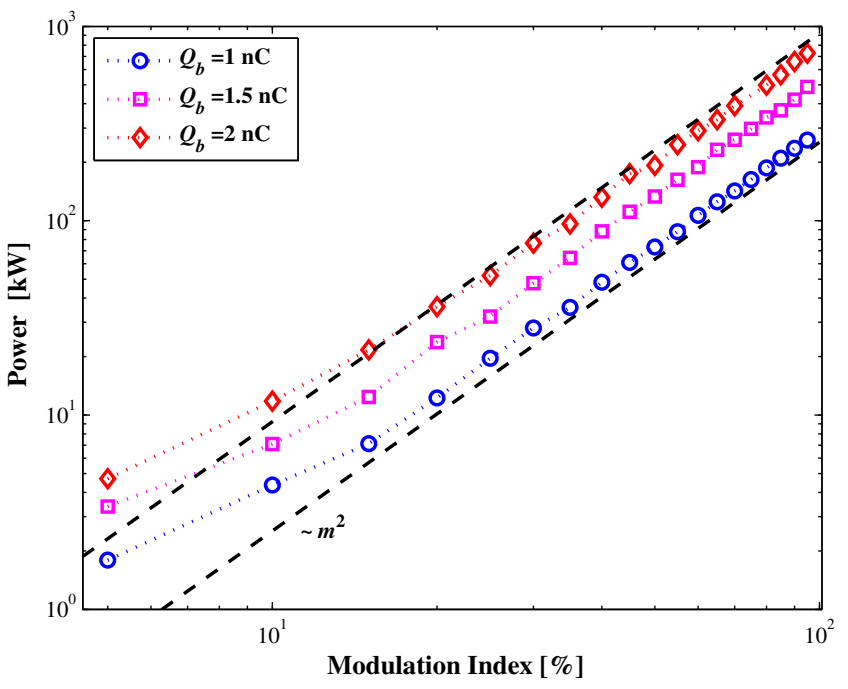

FIG. 8. Prebunched FEL radiation power as function of the modulation index $m$. The modulation frequency is $f_{m}=3 \mathrm{THz}$.

Dependence of the radiation spectrum and total power emitted on the modulation frequency is shown at Fig. 7. An optimal value of $f_{m}=3 \mathrm{THz}$ (which is close to the synchronism frequency $f_{s} \approx 2.96 \mathrm{THz}$ of the FEL configuration) was chosen for the following simulations. Figures 8 and 9 demonstrate the dependence of the total radiation power on the modulation index $m$ and the beam pulse charge, respectively. Beginning with the modulation of approximately 15\% depth (for the power as a function of the modulation depth) and up to the beam pulse charge of $Q_{b} \approx 1 \mathrm{nC}$ (for the power as a

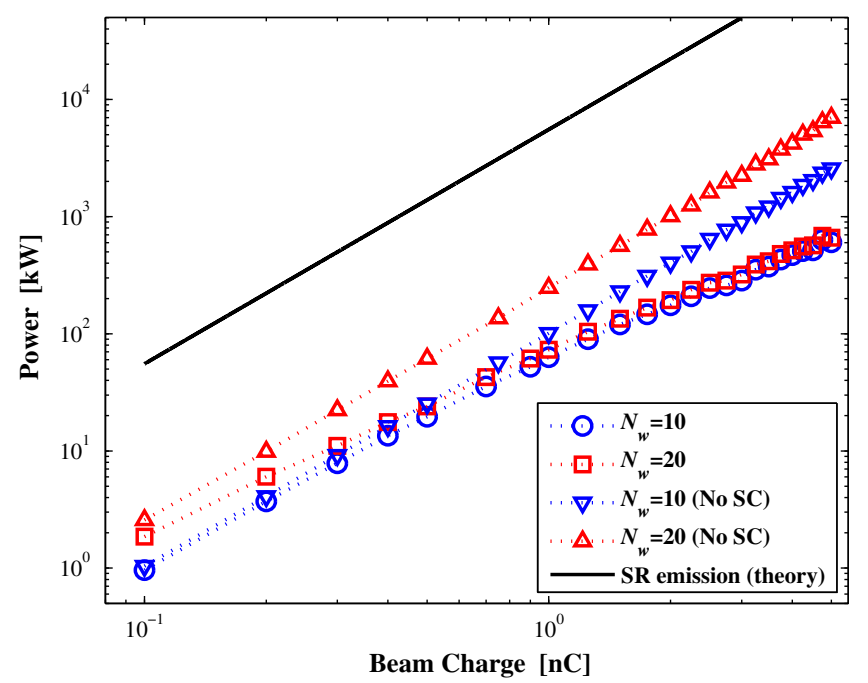

FIG. 9. Prebunched FEL radiation power as function of the beam pulse charge. The modulation index and the modulation frequency are $m=50 \%$ and $f_{m}=3 \mathrm{THz}$, respectively. 

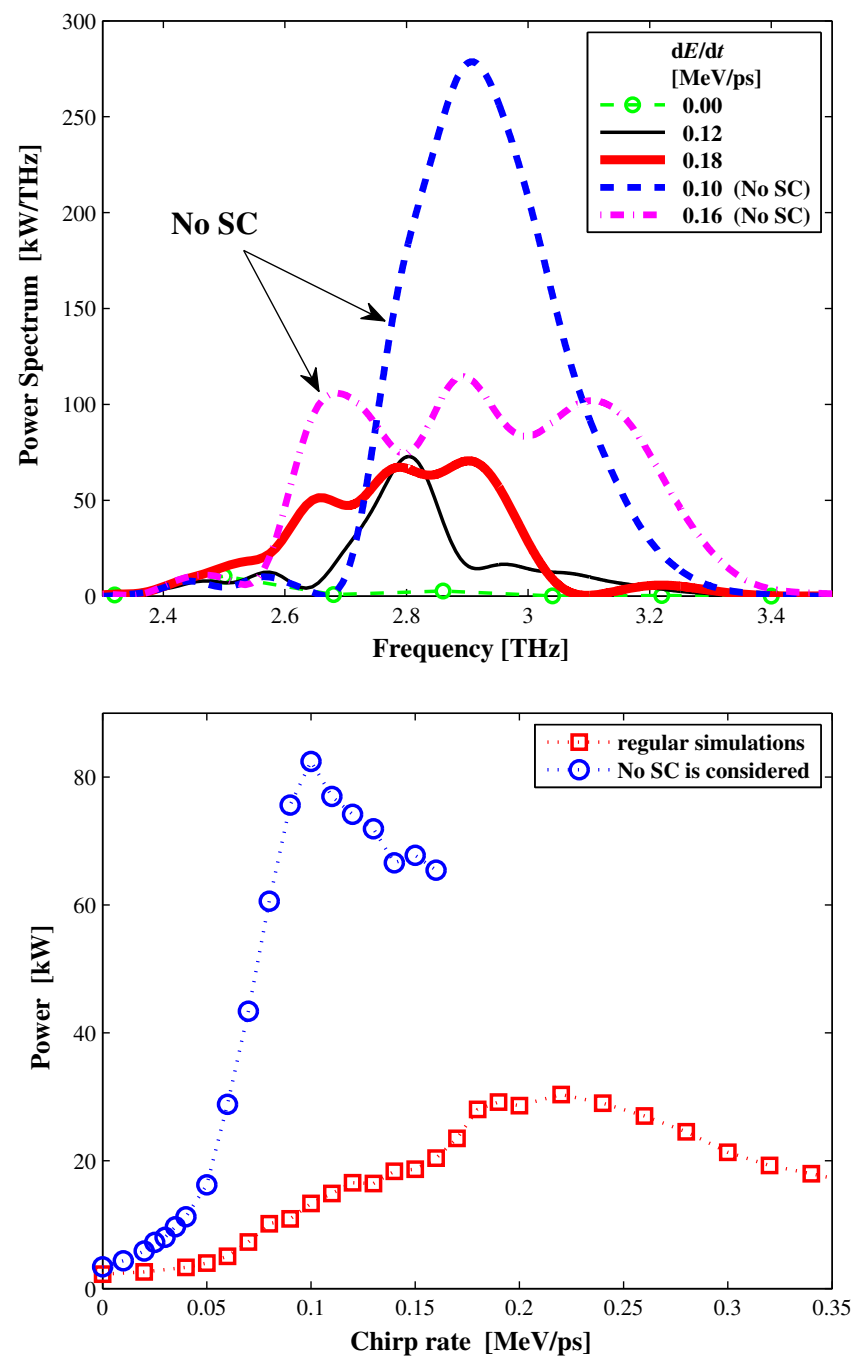

FIG. 10. Energy chirping of a $N_{m}=11$ long premodulated beam: radiation power spectrum (top), and the total emitted power as function of the chirp rate (bottom). On both pictures, results of the simulations with the SC forces "turned off" are shown for comparison. The beam charge is $Q_{b}=1 \mathrm{nC}$, the initial modulation depth and frequency are $m=50 \%$ and $f_{m}=2.5 \mathrm{THz}$, respectively.

function of the beam charge), the radiation power seems to be proportional to the modulation index squared and to the beam pulse charge squared, in close correspondence with the theory. Note that at higher beam pulse charges, the power grows with the beam charge is slowed due to spacecharge effects. To demonstrate this, results of simulations with the space-charge interaction "turned off" are also given for the comparison. Note that due to the destructive effect of space-charge forces, most of the radiation is emitted at the first part of the beam trajectory, so as the powers of the radiation emitted after the first 10 periods of the wiggler, and after all 20 periods are almost the same. In contrast, "turning off" the space-charge fields in the simulations enables a continuous growth of the

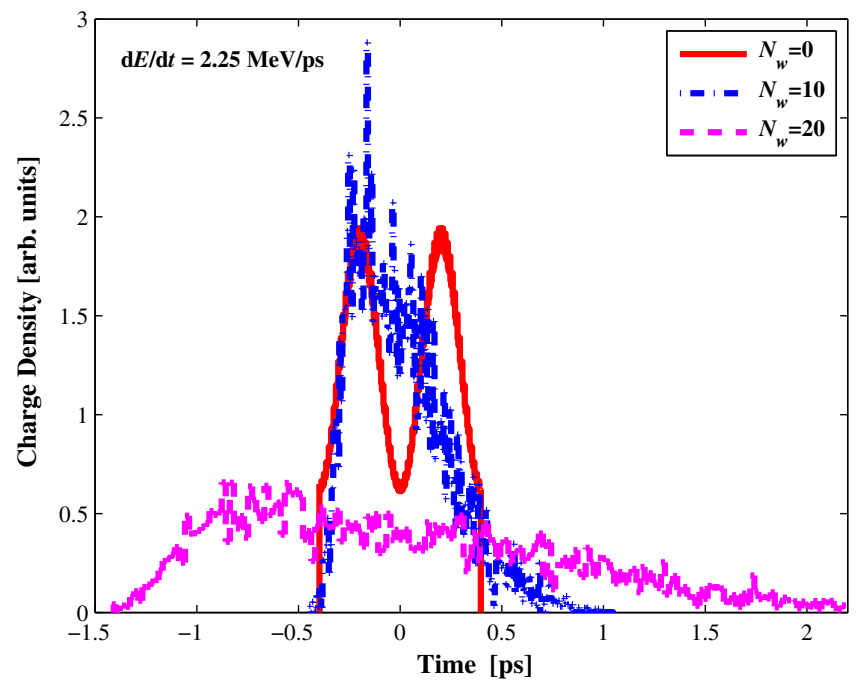

FIG. 11. Charge density of a $N_{m}=2$ long premodulated, energy chirped (the chirp rate of $d E / d t=2.25 \mathrm{MeV} / \mathrm{ps}$ ) beam pulse at the entrance (solid line), and after $N_{w}=10$ (dot-dashed line) or after $N_{w}=20$ (dashed line) wiggler's periods. The beam charge is $Q_{b}=1 \mathrm{nC}$, the initial modulation depth and frequency are $m=50 \%$ and $f_{m}=2.5 \mathrm{THz}$, respectively.

radiation power during the beam propagation along all the wiggler.

Energy chirping of premodulated beam pulses results in an increase of the beam modulation frequency along the beam propagation. Using such beam pulses, a considerable frequency up-shift can be obtained when a beam pulse modulated at a relatively low modulation frequency, radiates at a much higher FEL synchronism frequency. Moreover, energy chirping is believed to introduce some compensation of space-charge fields effecting the beam pulse, enabling a more efficient radiation emission. Figure 10 demonstrates the spectrum and the total power of the radiation emitted in such FEL structure driven by $N_{m}=11$ long premodulated beam pulse with an energy chirping. As clearly seen from the pictures, energy chirping of a beam pulse modulated at $f_{m}=2.5 \mathrm{THz}$ frequency provides at the chirp rate of about $0.22 \mathrm{MeV} / \mathrm{ps}$ (the maximum relative energy deviation of $\Delta E / E \approx 15 \%$ ) much stronger radiation then it is if no chirping is applied. The radiation spectrum covers a wide frequency range of about $0.5 \mathrm{THz}$ bandwidth, what can be useful in some applications. Effects of space-charge interactions are demonstrated by a comparison with the corresponding results obtained with space-charge forces "turned off" in the simulations.

For a given beam energy, the chirp rate is limited because the radiation of different electrons in the beam should provide a positive interference each other along the beam trajectory; therefore the maximum relative energy deviation should be small: $\Delta E / E=\left(\frac{d E}{d t}\right) \cdot \frac{m}{E_{b} f_{m}} \ll 1$ to let the electrons of the beam propagate along the wiggler as a single 

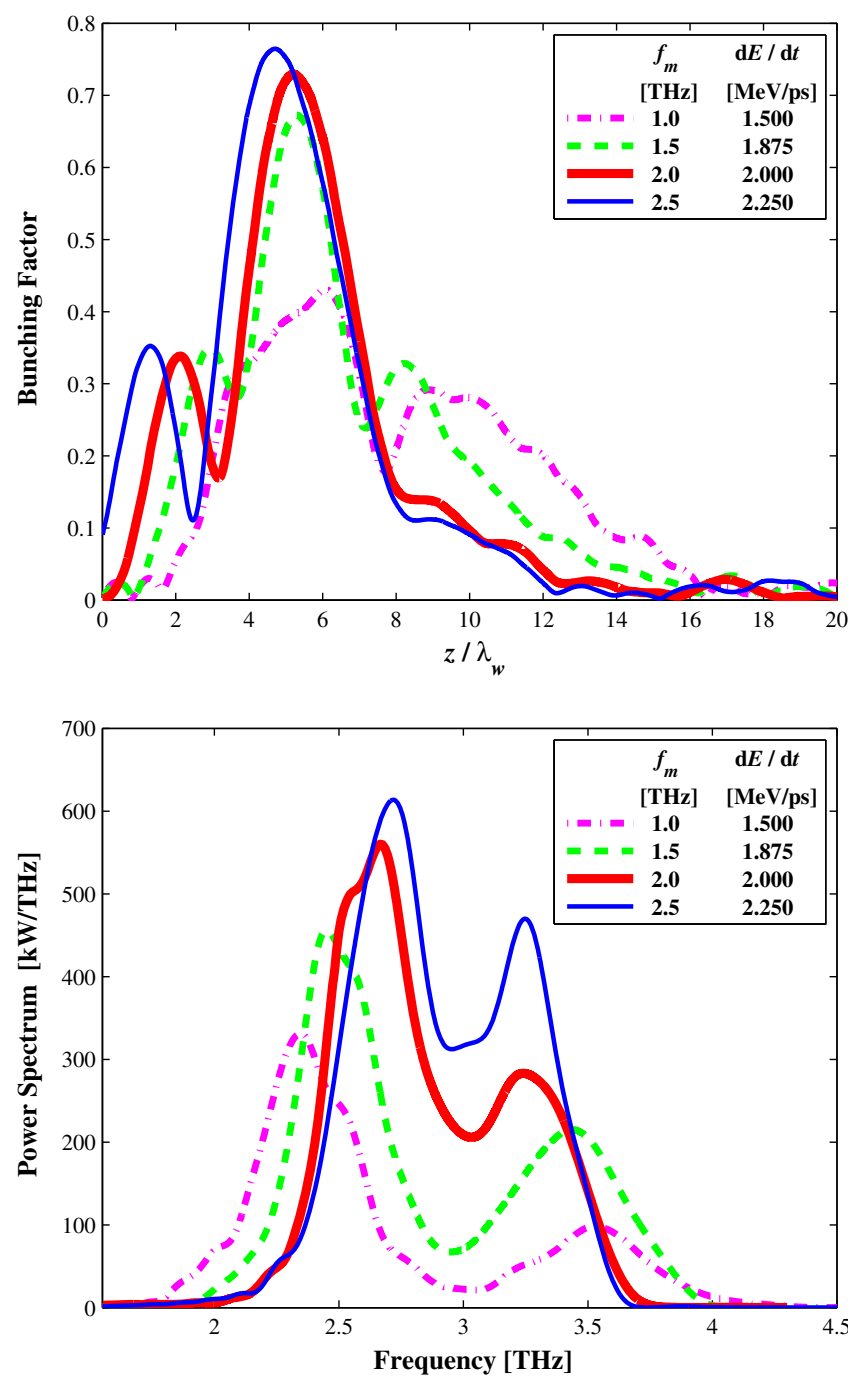

FIG. 12. The bunching factor $\left\langle\exp \left(j w_{s} t\right)\right\rangle$ at the FEL synchronism frequency (top) and the power spectrum (bottom), obtained with short $N_{m}=2$ long premodulated, energy chirped beam pulses. The beam charge is $Q_{b}=1 \mathrm{nC}$, the initial modulation depth is $m=50 \%$.

bunch. Higher chirp rates can be applied if a shorter beam pulse is used, and stronger effects are obtained in this case. The use of short beam pulses (for example, consisting just of a couple modulation periods $N_{m}=2$ ) with relatively high chirping rates, seems to enable an effective FEL scheme. Energy chirping of such pulses can give rise to a formation of a single, ultra short peak, as demonstrated in Fig. 11. This density compression provides a high bunching and a more strong coherent radiation emission, as shown in Fig. 12. The results shown were obtained with different initial modulation frequencies (from 1.0 to $2.5 \mathrm{THz}$ ); an "optimal" chirp rate was chosen for each modulation frequency according to results presented at Fig. 13, where the radiation power is given in dependence on the chirp rate.

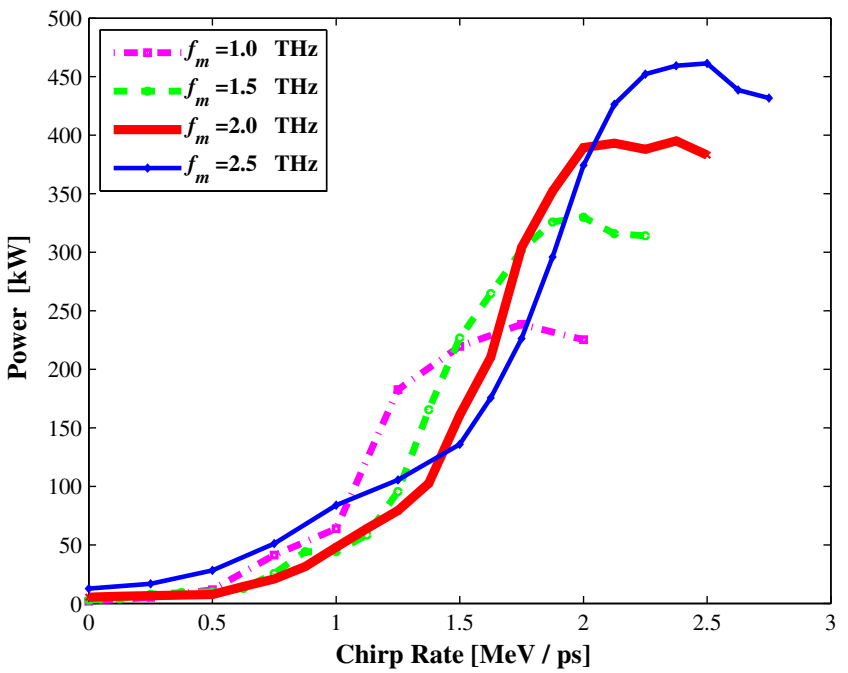

FIG. 13. Radiation power of prebunched FEL driven by a $N_{m}=$ 2 long premodulated beam pulse with energy chirping. The beam charge is $Q_{b}=1 \mathrm{nC}$, the initial modulation depth is $m=50 \%$.

\section{CONCLUSIONS}

Two possible configurations of a single pass, $\mathrm{THz}$ spectral range free-electron laser driven by a photocathode hybrid rf-LINAC are discussed in the present work: an enhanced coherent spontaneous emission FEL, and a prebunched FEL utilizing periodically modulated short electron beam pulses. A general study of the FEL configurations is carried out in the framework of the spacefrequency approach, realized in WB3D numerical code. The configurations are studied and compared based on preliminary parameters of the drive hybrid rf-LINAC gun being currently built in University of California, Los Angeles for the Israeli THz FEL project developed in Ariel University.

ECSE FEL driven by short (a sub-picosecond or a femtosecond scale long) electron beam pulses with a controlled energy chirp can be produced by means of a photocathode hybrid rf-LINAC [15] by some phase desynchronization of electron pulses accelerated preliminary in the standing wave part of the hybrid gun, relative to the electromagnetic wave in the following traveling wave cavity. Such operation of the united hybrid electron gun seems to be similar to application of the "phase-matching" section within a FEL beam line utilized in [3]. At a proper chirp rate, enhanced CSE is found to provide a beam bunching of more than 75-80\%. This promises a high efficiency of such radiation source. Unfortunately, destructive space-charge forces emerge to play an important role and to restrict efficiency of ECSE FEL if overcharged bunches are applied. Nevertheless, Tera-Hertz radiation with the peak power of about $36-38 \mathrm{~kW}$ (the energy flux of approximately $0.5 \mu \mathrm{J}$ at the radiation extraction efficiency of $\sim 0.04 \%$ ) is found to be achievable in the ECSE FEL configuration considered with realistic beam pulse charges 
of $Q_{b}=300 \mathrm{pC}$. Note that for ECSE FEL, the radiation extraction efficiency scales about linearly with the beam pulse charge.

Splitting of the photocathode's optical laser beam with following interference of resulted partial beams on the photocathode enables production of modulated beam pulses of a desired modulation frequency in the TeraHertz frequency range, and even provides a control on a number of modulation oscillations [4]. These electron beam pulses could drive a prebunched beam FEL.

Relatively long (a few picosecond long), monoenergetic beam pulses or beam pulses with a low energy chirping can be utilized in this FEL configuration. Using longer beam pulses (in comparison with those used in ECSE FEL discussed before) results in reduction of destructive space-charge interactions, enabling the use of higher beam charges (up to a few nano-Coulomb). Thus, providing a stronger radiation emission (radiation with power of about $180-190 \mathrm{~kW}$ and energy flux of $\sim 1.2 \mu \mathrm{J}$ can be achieved in this scheme with $2 \mathrm{nC}$ beam pulse charge, what corresponds to the radiation extraction efficiency of $\sim 0.01 \%$ ) in comparison with the previous FEL configuration.

Another concept of prebunched FEL shortly discussed here includes the use of short (a couple of the modulation periods long $N_{m}=2$ ) electron pulses with a high energy chirping. Density compression of short beam pulses produces a strong "pressure" on the electrons in the bunch center, giving rise for while to the formation of an ultrashort, femtoscale long electron pulse. A high bunching of $70-75 \%$ resulted explaining a wide band, powerful undulator radiation obtained with these pulses, which for the beam pulse charge of $Q_{b}=1 \mathrm{nC}$ is characterized by the bandwidth of about a one Giga-Hertz, and a peak power of $0.2-0.45 \mathrm{MW}$ (energy flux of 1.4-3.0 $\mu \mathrm{J}$, and the radiation extraction efficiency of $\sim 0.05 \%$ ). More detailed study of this FEL configuration should be conducted in the future.

Both ECSE and prebunched FEL configurations demonstrate a high effectiveness at the parameters range under the consideration, and are supposed to be realized experimentally in the framework of the Israeli rf-LINAC THz FEL project developed in Ariel university.

[1] E. Dyunin, Yu. Lurie, Y. Pinhasi, A. Gover, and H. Marks, in IEEE 25th Convention of Electrical and Electronics Engineers in Israel, 2008 (IEEE, Eilat, 2008), p. 825; A. Friedman, N. Balal, E. Dyunin, Yu. Lurie, E. Magori, V. L. Bratman, J. Rosenzweig, H. Lay To, and A. Gover, in Proceedings of the 36th International Free Electron Laser Conference, Basel, Switzerland, 2014 (JACoW, Basel, 2014), p. 553, http://accelconf.web.cern.ch/AccelConf/ FEL2014/papers/tup081.pdf.

[2] Yu. Lurie and Y. Pinhasi, Enhanced super-radiance from energy-modulated short electron bunch free-electron lasers, Phys. Rev. ST Accel. Beams 10, 080703 (2007).
[3] A. Doria, G. P. Gallerano, E. Giovenale, S. Letardi, G. Messina, and C. Ronsivalle, Enhancement of Coherent Emission by Energy-Phase Correlation in a Bunched Electron Beam, Phys. Rev. Lett. 80, 2841 (1998); A. Doria, V. B. Asgekar, D. Esposito, G. P. Gallerano, E. Giovenale, G. Messina, C. Ronsivalle, Long wavelength compact-FEL with controlled energy-phase correlation, Nucl. Instrum. Methods Phys. Res., Sect. A 475, 296 (2001); A. Doria, G. P. Gallerano, E. Giovenale, G. Messina, and I. Spassovsky, Enhanced Coherent Emission of Terahertz Radiation by Energy-Phase Correlation in a Bunched Electron Beam, Phys. Rev. Lett. 93, 264801 (2004).

[4] Y. Shen, Xi Yang, G. L. Carr, Y. Hidaka, J. B. Murphy, and $\mathrm{X}$. Wang, Tunable Few-Cycle and Multicycle Coherent Terahertz Radiation from Relativistic Electrons, Phys. Rev. Lett. 107, 204801 (2011).

[5] Y. Pinhasi, Yu. Lurie, and A. Yahalom, Space-frequency model of ultra wide-band interactions in free-electron lasers, Phys. Rev. E 71, 036503 (2005).

[6] Y. Pinhasi, Yu. Lurie, and A. Yahalom, Model and simulation of wide-band interaction in free-electron lasers, Nucl. Instrum. Methods Phys. Res., Sect. A 475, 147 (2001).

[7] Y. Pinhasi and Yu. Lurie, Generalized theory and simulation of spontaneous and super-radiant emissions in electron devices and free-electron lasers, Phys. Rev. E 65, 026501 (2002).

[8] Y. Pinhasi, Yu. Lurie, A. Yahalom, and A. Abramovich, Space-frequency model of amplified spontaneous emission and super-radiance in free electron laser operating in the linear and non-linear regimes, Nucl. Instrum. Methods Phys. Res., Sect. A 483, 510 (2002).

[9] Y. Pinhasi, Y. Lurie, and A. Yahalom, Study of radiation spectrum in a free electron laser oscillator from noise to saturation, Nucl. Instrum. Methods Phys. Res., Sect. A 528, 62 (2004).

[10] A. Gover, E. Dyunin, Y. Lurie, Y. Pinhasi, and M. V. Krongauz, Superradiant and stimulated - Superradiant emission in pre-bunched electron-beam radiators. II: Radiation enhancement schemes, Phys. Rev. ST Accel. Beams 8, 030702 (2005).

[11] Yu. Lurie and Y. Pinhasi, Modal description of longitudinal space-charge fields in pulse-driven freeelectron devices, Phys. Rev. ST Accel. Beams 13, 050701 (2010).

[12] V. Zhaunerchyka, R. T. Jongma, Yu. Lurie, Y. Pinhasi, and W. J. van der Zande, Selective amplification of the lowerfrequency branch via stimulated super-radiance in a waveguided free electron laser oscillator driven by short electron bunches, Appl. Phys. Lett. 97, 231109 (2010).

[13] B. W. J. McNeil, M. W. Poole, and G. R. M. Robb, Unified model of electron beam shot noise and coherent spontaneous emission in the helical wiggler free electron laser, Phys. Rev. ST Accel. Beams 6, 070701 (2003).

[14] http://www.pulsar.nl/gpt/.

[15] J. Rosenzweig et al., Design and applications of an X-band hybrid photoinjector, Nucl. Instrum. Methods Phys. Res., Sect. A 657, 107 (2011). 